\title{
Review Article \\ Diversity in the Regulation of Autophagy and Mitophagy: Lessons from Parkinson's Disease
}

\author{
Charleen T. Chu \\ Division of Neuropathology, Department of Pathology, University of Pittsburgh School of Medicine, Pittsburgh, PA 15261, USA
}

Correspondence should be addressed to Charleen T. Chu, ctc4@pitt.edu

Received 23 November 2010; Accepted 22 January 2011

Academic Editor: David K. Simon

Copyright (C) 2011 Charleen T. Chu. This is an open access article distributed under the Creative Commons Attribution License, which permits unrestricted use, distribution, and reproduction in any medium, provided the original work is properly cited.

\begin{abstract}
Selective mitochondrial degradation through autophagy (mitophagy) has emerged as an important homeostatic mechanism in a variety of organisms and contexts. Complete clearance of mitochondria can be observed during normal maturation of certain mammalian cell types, and during certain forms of neuronal cell death. In recent years, autophagy dysregulation has been implicated in toxin-injured dopaminergic neurons as well as in major genetic models of Parkinson's disease (PD), including $\alpha$-synuclein, leucine-rich repeat kinase 2 (LRRK2), parkin, PTEN-induced kinase 1 (PINK1), and DJ-1. Indeed, PINK1-parkin interactions may form the basis of a mechanism by which dissipation of the inner mitochondrial membrane potential can trigger selective mitochondrial targeting for autophagy. Multiple signals are likely to exist, however, depending upon the trigger for mitophagy. Similarly, the regulation of basal or injury-induced autophagy does not always follow canonical pathways described for nutrient deprivation. Implications of this regulatory diversity are discussed in the context of neuronal function and survival. Further studies are needed to address whether alterations in autophagy regulation play a directly injurious role in PD pathogenesis, or if the observed changes reflect impaired, appropriate, or excessive autophagic responses to other forms of cellular injury.
\end{abstract}

\section{Introduction}

Macroautophagy represents an evolutionarily conserved response to nutrient stresses, which also plays an increasingly recognized role in basal cellular maintenance and in cellular responses to injury. The important role of macroautophagy in brain development and quality control was highlighted by observations that mice engineered for deficiency in key autophagy genes exhibit spontaneous neurodegeneration with ubiquitinated protein aggregates [1, 2]. Macroautophagy (hereafter, autophagy unless otherwise specified) is also implicated in mitochondrial quality control, as altered mitochondria accumulate under basal conditions of autolysosomal dysfunction [3-6], and mitophagy is further induced in cells exhibiting damaged mitochondria $[7,8]$. Mitochondrial autophagy induced in response to mitochondrial damage or neuronal injury may play either prosurvival $[7,9]$ or prodeath roles $[10,11]$. As mechanisms underlying different models of injury-induced autophagy and mitophagy are discovered, the concept of distinct regulatory inputs to a core autophagy pathway has emerged.

In sympathetic neurons, it was noted that the phosphoinositide 3-kinase inhibitor 3-methyladenine (3-MA) delayed apoptosis by reducing cytochrome $\mathrm{c}$ release and caspase activation [12]. Subsequent studies in the same system resulted in the first report of complete and selective clearance of mitochondria from neurons [13]. Combined with developmental observations of selective mitochondrial clearance in reticulocytes, lens, and lymphocytes [14, 15], these findings implicate the existence of specific mechanisms targeting mitochondria for autophagic clearance (although nonautophagic mechanisms may also contribute $[16,17])$. Autophagy dysregulation is observed in a growing number of toxic/environmental and genetic models of Parkinson's disease (PD). Recent breakthroughs show a key role for two recessive Parkinsonian genes, PINK1 and parkin, in the specification of depolarized mitochondria for sequestration in aggresomes and/or autophagosomes [18, 19]. The potential 
role of autophagy in PD models is reviewed in relation to diverse regulatory pathways feeding into the core autophagy machinery.

\section{Autophagy Dysregulation in Parkinson's Disease}

2.1. Sporadic PD. In PD patient tissues, evidence of apoptosis and of autophagy is observed in substantia nigra neurons [20]. Additional studies demonstrate mitophagy in the substantia nigra neurons of patients with PD spectrum diseases [21], and in Alzheimer's disease [22]. The ultrastructural observations of mitophagy in PD are correlated with a peculiar punctate/vesicular staining pattern for phosphorylated extracellular signal-regulated protein kinases (ERK1/2) [23, 24]. Although there have not been quantitative ultrastructural studies of autophagolysosomal structures in PD tissues, analysis of punctate/vesicular phospho-ERK1/2 indicates preferential involvement of the substantia nigra ventrolateral tier [21]. Incidental Lewy body disease, which is thought to represent a preclinical form of $\mathrm{PD}$, exhibits an intermediate level of involvement [21]. In contrast, substantia nigra dopaminergic neurodegeneration associated with a distinct disease, progressive supranuclear palsy, do not show these changes (author's unpublished data), suggesting specificity for synucleinopathies.

2.2. Toxin Models of PD. Autophagy has been implicated in neurotoxin and environmental toxin models of dopaminergic cell death. Early and late autophagosomes can be identified by ultrastructural analysis, or by monitoring autophagosome-associated microtubule-associated protein 1 light chain 3 (LC3). Cytosolic LC3 migrates as an LC3-I band; upon stimulation of autophagy, LC3 is covalently conjugated to phospholipids, resulting in a faster migrating LC3-II band and correlating with punctate redistribution of the LC3 immunofluorescent signal. Increased autophagosomes have been described in acute injury models involving methamphetamine $[25,26]$, high doses of dopamine [27], 1-methyl-4-phenylpyridinium (MPP+) [10], 6-hydroxydopamine [11], the environmental toxicants rotenone [28], and paraquat [29]. In the case of toxins that are weak bases, it is not clear whether increased autophagosomes reflect increased autophagy induction or impaired completion of autophagic degradation from lysosomal $\mathrm{pH}$ elevation. Moreover, the role of autophagy in cell survival and cell death has been model-dependent. In some cases the "autophagy inhibitor" 3-MA exacerbated cell death [26] and autophagy stimulation conferred protection [28], but in other cases 3-MA ameliorated cell death $[27,30]$.

3-MA is a phosphoinositide 3-kinase (PI3K) inhibitor, which acts to inhibit autophagy by blocking the activity of the beclin 1-Vps34/class III PI3K complex. Interpretation of increased cell death in the presence of 3-MA, however, is complicated due to the ability of 3-MA to inhibit not only the class III PI3K involved in beclin 1-dependent autophagy pathways, but also the neuronal survival kinase Akt that is downstream of class I PI3K [12]. Due to opposite effects of class I and class III PI3Ks on autophagy [31], 3-MA can reduce or promote autophagy depending on the relative activation state of the two pathways [32]. 3-MA also has direct effects on glucose/glycogen metabolism independent of its autophagy-modulating effects and elevates lysosomal $\mathrm{pH}$ in living hepatocytes, but not isolated lysosomes [33]. Thus, it is essential to verify that 3-MA inhibits autophagy in the particular experimental condition being studied, and confirmation using more selective molecular inhibition of autophagy may be preferable.

While neuroprotective effects of autophagy in toxin models have been correlated with $\alpha$-synuclein sequestration [26], noncanonical beclin 1-independent autophagy/mitophagy contributes to MPP+ toxicity, as shown by RNA interference knockdown of Atg7 and LC3/Atg8 [10]. This implicates excessive activation of autophagy, since the participation of beclin 1 in autophagy is downregulated by binding to $\mathrm{Bcl}-2$ [34] or rubicon [35, 36]. Blunting the autophagy response has also been shown using dominant negative Vps34 to prevent hydrogen peroxide-mediated lysosomal leakage and caspase activation [37]. Likewise, a compound that activates mTOR to suppress autophagy confers protection from oxidative stress in neurons [30] while rapamycin exacerbates toxicity in this system and in primary neurons treated with $\mathrm{MPP}+[38]$.

2.3. Genetic Models of PD. The dominant PD-linked protein $\alpha$-synuclein exhibits consensus motifs for lysosomal degradation by chaperone-mediated autophagy (CMA), which is distinct from macroautophagy in its regulation. Mutant forms of $\alpha$-synuclein bind the CMA receptor but are not internalized, inhibiting this degradative pathway in isolated liver lysosomes [39]. Moreover, dopamine-oxidized forms of $\alpha$-synuclein show the same effect [40]. Both CMA and macroautophagy are involved in degrading wildtype $\alpha$-synuclein in neurons [41], and A53T $\alpha$-synuclein expression impairs CMA in living cells [42]. While upregulation of macroautophagy can mediate clearance of $\alpha$ synuclein aggregates in metabolically intact cells [43], reports that $\alpha$-synuclein can affect mitochondrial metabolism [44] and macroautophagy efficiency [45] raise additional questions. Indeed, CMA impairment induces upregulation of macroautophagy, which appears to contribute to neuron cell death [42]. Interestingly, low-dose application of the fusion inhibitor bafilomycin can protect against $\alpha$-synuclein pathology in C. elegans [46]. The reciprocal cross-regulation of autophagy and of $\alpha$-synuclein complicates analysis and creates the possibility of damaging feed-forward cycles.

One of the prominent phenotypes attributed to the dominant and sporadic PD-implicated protein LRRK2 is modulation of the neuritic arbor. Increased LRRK2 activity and PD-linked LRRK2 mutants cause simplification and shortening of neuritic projections while knockdown of LRRK2 expression results in enhanced neuritogenesis [47]. LRRK2-G2019S elicits neuritic autophagy, which mediates neurite shortening in retinoic acid-differentiated SH-SY5Y cells [48] and in primary cortical neurons [49]. LRRK2 associates with multivesicular bodies, and LRRK2-R1441G 
elicits increased autophagosomes attributed to disrupted autophagic flux in HEK-293 cells [50]. Whether cell type differences or somatic versus neuritic differences affect flux responses to mutant LRRK2 remain to be established, as estimates of autophagy induction and flux rates are inferred unless pulse-chase techniques are used.

Parkin deficiency causes different phenotypes in different model systems. In parkin knockout mice, the primary defect relates to neurotransmission [51, 52]. In Drosophila, however, prominent mitochondrial degeneration in flight muscles and sperm is observed [53]. A pivotal discovery for parkin function was made in HeLa cells treated with the mitochondrial depolarizing agent FCCP or CCCP [18]. Parkin translocation to FCCP-depolarized mitochondria results in their eventual clearance through Atg5-dependent mechanisms, and this observation has led to an explosion of papers on the subject, each of which sheds additional insight into molecular mechanisms of mitochondrial cargo specification (discussed below). While overexpressed parkin enhances mitophagy in FCCP-treated cells [18] and in PINK1-deficient cells [7], the role of endogenous parkin in this setting is less clear. Translocation of tagged parkin to mitochondria and its ubiquitinating activity is essential for enhanced mitochondrial autophagy in FCCP/CCCPtreated cells. However, parkin monoubiquitination of $\mathrm{Bcl} 2$ enhances the ability of $\mathrm{Bcl} 2$ to bind beclin 1 and suppress autophagy, and RNAi knockdown of parkin increases the LC3-II band in 293, SH-SY5Y, and primary neuron cultures [54]. Thus, depending on subcellular localization and/or target accessibility, parkin can act to either promote mitochondrial specification for autophagy or to downregulate general autophagy.

PINK1 knockdown cells exhibit mitochondrial functional and morphological abnormalities [7, 55-57], with enhanced autophagic clearance of mitochondria [7]. On the other hand, overexpressed, full-length PINK1 reduces unconjugated LC3 [58] and increases parkin localization to mitochondria ([59,60] and discussed below). Endogenous PINK1 in SH-SY5Y cells is predominantly processed [7], and PINK1 is processed in Drosophila by the membrane protease Rhomboid-7 [61]. As mitochondrial protein import and processing depends upon an intact inner mitochondrial membrane potential, stabilization of full-length PINK1 at the surface of depolarized mitochondria initiates PINK1dependent mitophagy enhancement $[62,63]$.

Mitochondrial dysfunction observed in DJ-1 null cells is accompanied by a baseline decrease in the activated LC3-II band $[6,64]$. However, whether this reflects increased or decreased autophagic flux remains controversial, and an increase in markers of compensatory mitophagy was recently reported in DJ-1 shRNA-expressing neuroblastoma cells [65]. DJ-1 null fibroblasts show reductions in expression of rapamycin-induced autophagosome markers in one study, interpreted as indicative of decreased autophagic induction [6]. Based on decreased basal levels of the autophagy substrate and cargo adaptor p62, however, another study concluded increased autophagic flux [64]. Flux analysis of autophagy or mitophagy can be technically challenging, but it is also possible that DJ-1 has different effects on basal versus induced autophagy. Interestingly, DJ-1 null cells exhibited decreased phosphorylation of ERK1/2 [6], which mediates autophagy/mitophagy in several systems $[10,11$, $48,66,67]$. DJ-1 siRNA has also been reported to inhibit paraquat-induced autophagy [68].

\section{Diversity in the Regulation of Autophagy}

3.1. Canonical Pathway of Starvation-Induced Autophagy. The identification of yeast genes necessary for autophagy, and related membrane trafficking events revolutionized the study of mitophagy in health and disease [69]. In brief, amino acid signals and insulin signals converge in turning on the mammalian target of rapamycin (mTOR), which suppresses autophagy. Amino acids also suppress ERK1/2 signaling. Loss of insulin signals, loss of amino acids, or direct inhibition of mTOR then serve to derepress autophagy induction, while $5^{\prime}$ adenosine monophosphateactivated protein kinase (AMPK) senses low energy to turn on autophagy. Beclin 1-Vps34-mediated changes in lipid composition are needed to define the phagophore and nucleate the membrane deposition of ubiquitin-like proteins Atg12 and LC3 in response to deprivation of growth factors or nutrients. Because beclin 1 can be found in several competing protein complexes [34-36], beclin 1-dependence has been proposed to serve a potential rheostat role in fine tuning levels of autophagy.

3.2. Mitophagy Regulation during Nutrient-DeprivationInduced Autophagy. Starvation-induced autophagy is traditionally thought of as a nonselective bulk degradation process, with nonselective or bystander engulfment of mitochondria. However, yeast studies suggest a degree of mitochondrial recognition even in this process. The clearance of presumably undamaged mitochondria during nitrogen starvation requires the presence of an outer mitochondrial membrane protein Uth1p, [70], which does not have a clear mammalian homolog. Efficient mitochondrial autophagy in stationary-phase yeast are also regulated by an intermembrane space protein Aup1p [71]. Yeast cells grown in lactate undergo mitochondrial autophagy. Recently, the mitochondrial protein Atg32 was identified as a yeast mediator of selective mitophagy [72]. Atg 32 binds to Atg11, a known adaptor protein for selective autophagy in yeast. This system recruits mitochondria to autophagosomes, but does not directly regulate macroautophagy induction itself.

3.3. Beclin 1-Independent Injury-Induced Autophagy. Mitophagy is induced in neuronal cells and primary neurons injured with MPP+. Interestingly, in this system, autophagy induction proceeds even in the presence of PI3K inhibitors or siRNA knockdown of beclin 1 [10]. PI3K inhibitors are also unable to inhibit the selective clearance of photodamaged mitochondria in hepatocytes [73]. Beclin 1-independent mitophagy would no longer be negatively regulated by $\mathrm{Bcl} 2$ or rubicon, and thus, is more likely to allow a harmful level of autophagy activation. While the mechanism of beclin 1independent autophagy has not been defined, it could reflect 
alternative enzymatic means of increasing localized membrane concentrations of phosphatidylinositol 3-phosphate (PI(3)P) [38]. Alternatively, there could be other pathways of nucleation in which membrane changes mediated by ROS or kinase activation can substitute for $\mathrm{PI}(3) \mathrm{P}$ in recruiting Atg18-Atg2 [74] or Atg16L to membranes.

Beclin 1-independent autophagy has been described in several model systems. As inhibition of autophagy is protective in these models [10,48, 75-77], these observations support the concept of harmful overactivation of autophagy. It remains to be determined whether this is accidental, or forms part of a programmed cell death pathway.

3.4. Quality Control Autophagy and Cargo Regulation. Other variations from classic rapamycin-induced autophagy are beginning to emerge. Both basal autophagy and injuryinduced autophagy play roles in organellar and protein quality control. A key feature of quality control-related autophagy relates to the ability of the cellular autophagy machinery to selectively remove damaged proteins and organelles while sparing their normal counterparts.

Interestingly, studies of quality control autophagy reveal requirements for HDAC6 and actin remodeling for maturation and completion of autophagy [78]. HDAC6-dependent retrograde transport of autophagy substrates and mediators to the perinuclear region is necessary for degradation of aggregated huntingtin [79], and selective transport may represent one mechanism of cargo enrichment. For protein aggregates, another mechanism of cargo recruitment involves direct adaptor protein interactions mediated by p62, which bridges ubiquitin on the cargo with LC3 on the autophagic membrane $[80,81]$. Other adapter proteins that have been identified include NBR1, which can cooperate with p62 [82], and Nix, which binds to GABARAP-L1 [83]. While specific proteins may differ between yeast and mammals, the general concept of cargo receptors and adapter proteins that link into the autophagy machinery represents a rapidly emerging area of research.

3.5. Depolarization-Induced Mitophagy. One of the exciting developments in mitophagy regulation is the use of chemically depolarized mitochondria to dissect proteins needed for mitochondrial clearance. With nutrient deprivation, depolarization of mitochondria can occur after sequestration by GFP-LC3 [84], preceding their entry into acidic lysosomal compartments in rat hepatocytes [85]. Live imaging studies also show that mitotracker-labeled mitochondria disappear within 8 minutes of entering lysotracker-stained lysosomes in hepatocytes [86]. Thus, the observation of "mitophagosomes" is a transient event, most readily observed when elicited by synchronized chemical insults, or with inhibition of autophagosome maturation [11,87].

The past year has witnessed significant advances in delineating mechanism(s) by which depolarization promotes mitophagy, subsequent to the original observation that the ubiquitin ligase parkin translocates to mitochondria in FCCP/CCCP-treated cells to mediate their clearance [18]. Of note is the observation that parkin functions only in the cargo recruitment step of mitophagy, but other mechanisms involving Nix mediate the induction of autophagy by depolarization [8]. Subsequent discussion will focus on surface changes on depolarized mitochondria that could mediate their autophagic recruitment in the depolarization model.

Depolarization inhibits membrane potential-dependent proteolytic processing of PINK1 [88]. This, in turn, causes full-length PINK1 to accumulate at the surface of mitochondria $[62,63]$, which is necessary for stable and global association of parkin with mitochondria in the FCCP/CCCP treated cells. From here, several mechanisms have been described that could specify parkin-bearing mitochondria for mitophagy. Parkin ubiquitination of mitofusins serve to promote fission $[60,89]$, which is necessary for mitophagy [87]. Parkin has also been reported to polyubiquitinate voltage-dependent anion-selective channel protein 1 (VDAC1) [90], which may explain PINK1-parkin-dependent perinuclear aggregation of mitochondria [19, 91]. VDAC1 interacts with a dynein light chain [92], although it is unknown whether or not ubiquitination modulates this. PINK1 may also interact directly with LC3 [59]. Finally, the p62 adaptor discussed above is recruited to parkinubiquitinated mitochondria, although its role in aggregation versus mitophagy recruitment is controversial $[90,93]$.

3.6. Alternative Mechanisms Relating to Mitophagy. Mitophagy can be initiated prior to depolarization of the mitochondrial membrane potential in several model systems. In starvation-induced mitophagy in hepatocytes, mitochondria remain polarized until after they are encircled by GFP-LC3 [84]. Likewise, mitochondrial depolarization occurs downstream of autophagosome formation in reticulocytes during Nix-dependent developmental mitophagy [94]. Indeed, depolarization-independent, Nix-dependent mitophagy may involve direct interactions of Nix with an LC3 homolog GABARAP [83], indicating alternative signaling for mitochondria recognition during autophagosome formation.

Parkin also compensates for PINK1 deficiency $[95,96]$. While this could be mediated by nonmitophagy-related mechanisms, increased parkin expression causes increased autophagy in PINK1-deficient cells [7], and parkin-mediated protection from cell death is substantially diminished by RNAi knockdown of autophagy mediators [9]. Whether or not stable parkin recruitment to mitochondria is necessary for its compensatory effects is unknown, but hydrogen peroxide can recruit parkin to mitochondria through a mechanism not requiring mitochondrial targeting of PINK1 [97]. Finally, a parkin mutant that is deficient in translocating to mitochondria can be rescued in this function by the DnaJ/Hsp40 chaperone HSJ1a [98]. Besides ubiquitination, phosphorylation or changes in lipid composition could conceivably also trigger loss of mitochondria [99].

It is reasonable to assume that these mechanisms would allow for selective removal of irreversibly damaged mitochondria while sparing normal mitochondria, but this has not yet been robustly demonstrated. FCCP- or CCCPtreated cell lines exhibit rather global changes of parkin 
translocation, perinuclear aggregation, and clearance. Likewise, selective autophagy of mitochondria with mtDNA mutations is inferred from studies showing that fibroblasts from patients with mitochondrial DNA diseases exhibit higher rates of mitophagy upon amino acid deprivation than those containing normal mitochondria [100], but selective autophagic engagement of individual mitochondria with high burdens of mtDNA mutations was not assessed. Cells expressing mitochondrial DNA deletions exhibit elevated transcripts for Atg proteins [101], and thus generalized elevations in autophagic capacity may also result in greater mitophagy for stochastic reasons. Live imaging studies such as those demonstrating that relatively depolarized mitochondria show decreased fusion [102], or preferential retrograde axonal transport [103], could be used to further establish selective recognition of abnormal mitochondria on an organelle-by-organelle basis.

\section{Summary}

Autophagy has emerged as a central response observed in multiple models of Parkinsonian neurodegeneration. In several chronic models, autophagy induction plays beneficial roles in clearing protein aggregates or damaged mitochondria. Autophagy can also play a harmful role in neurons subjected to acute injury such as ischemia-reperfusion or neurotoxin treatment. As understanding of mechanisms underlying autophagy and mitophagy develops, it will be interesting to determine whether distinct regulatory inputs to the core autophagy machinery underlies differences in the degree and outcome of autophagy or mitophagy induction. Just as pathways are emerging that show differences between autophagy induced for quality control and in response to trophic/nutrient deprivation, variations on the depolarization-initiated PINK1-parkin pathway are likely to emerge. Cell type specific mechanisms must also be considered, as parkin translocation is not observed in CCCP-treated cortical and striatal/midbrain neurons [104]. Redundancy in mechanisms that underlie the removal of damaged mitochondria may account for the relatively minor symptoms observed in knockout mice and form the basis for future therapies to heighten neuroprotective responses in PD patients.

\section{Acknowledgments}

Work in the author's laboratory is supported in part by the National Institutes of Health (AG026389, NS065789). C. T. Chu is recipient of an AFAR/Ellison Medical Foundation Julie Martin Mid-Career Award in Aging Research.

\section{References}

[1] M. Komatsu, S. Waguri, T. Chiba et al., "Loss of autophagy in the central nervous system causes neurodegeneration in mice," Nature, vol. 441, no. 7095, pp. 880-884, 2006.

[2] T. Hara, K. Nakamura, M. Matsui et al., "Suppression of basal autophagy in neural cells causes neurodegenerative disease in mice," Nature, vol. 441, no. 7095, pp. 885-889, 2006.
[3] Y. Zhang, H. Qi, R. Taylor, W. Xu, L. F. Liu, and S. Jin, "The role of autophagy in mitochondria maintenance: characterization of mitochondrial functions in autophagydeficient S. cerevisiae strains," Autophagy, vol. 3, no. 4, pp. 337-346, 2007.

[4] J. J. Jennings, J. H. Zhu, Y. Rbaibi, X. Luo, C. T. Chu, and K. Kiselyov, "Mitochondrial aberrations in mucolipidosis type IV," Journal of Biological Chemistry, vol. 281, no. 51, pp. 39041-39050, 2006.

[5] M. Komatsu, S. Waguri, T. Ueno et al., "Impairment of starvation-induced and constitutive autophagy in Atg7deficient mice," Journal of Cell Biology, vol. 169, no. 3, pp. 425-434, 2005.

[6] G. Krebiehl, S. Ruckerbauer, L. F. Burbulla et al., "Reduced basal autophagy and impaired mitochondrial dynamics due to loss of Parkinson's disease-associated protein DJ-1," PLoS ONE, vol. 5, no. 2, article e9367, 2010.

[7] R. K. Dagda, S. J. Cherra, S. M. Kulich, A. Tandon, D. Park, and C. T. Chu, "Loss of PINK1 function promotes mitophagy through effects on oxidative stress and mitochondrial fission," Journal of Biological Chemistry, vol. 284, no. 20, pp. 13843-13855, 2009.

[8] W.-X. Ding, H.-M. Ni, M. Li et al., "Nix is critical to two distinct phases of mitophagy, reactive oxygen species-mediated autophagy induction and Parkin-ubiquitin-p62-mediated mitochondrial priming," Journal of Biological Chemistry, vol. 285, no. 36, pp. 27879-27890, 2010.

[9] R. K. Dagda and C. T. Chu, "Mitochondrial quality control: insights on how Parkinson's disease related genes PINK1, parkin, and Omi/HtrA2 interact to maintain mitochondrial homeostasis," Journal of Bioenergetics and Biomembranes, vol. 41, no. 6, pp. 473-479, 2009.

[10] J. H. Zhu, C. Horbinski, F. Guo, S. Watkins, Y. Uchiyama, and C. T. Chu, "Regulation of autophagy by extracellular signal-regulated protein kinases during 1-methyl-4phenylpyridinium-induced cell death," American Journal of Pathology, vol. 170, no. 1, pp. 75-86, 2007.

[11] R. K. Dagda, J. Zhu, S. M. Kulich, and C. T. Chu, "Mitochondrially localized ERK2 regulates mitophagy and autophagic cell stress: implications for Parkinson's disease," Autophagy, vol. 4, no. 6, pp. 770-782, 2008.

[12] L. Xue, G. C. Fletcher, and A. M. Tolkovsky, "Autophagy is activated by apoptotic signalling in sympathetic neurons: an alternative mechanism of death execution," Molecular and Cellular Neurosciences, vol. 14, no. 3, pp. 180-198, 1999.

[13] L. Xue, G. C. Fletcher, and A. M. Tolkovsky, "Mitochondria are selectively eliminated from eukaryotic cells after blockade of caspases during apoptosis," Current Biology, vol. 11, no. 5, pp. 361-365, 2001.

[14] J. Zhang and P. A. Ney, "NIX induces mitochondrial autophagy in reticulocytes," Autophagy, vol. 4, no. 3, pp. 354356, 2008.

[15] H. H. Pua, J. Guo, M. Komatsu, and Y. W. He, "Autophagy is essential for mitochondrial clearance in mature T lymphocytes," Journal of Immunology, vol. 182, no. 7, pp. 4046-4055, 2009.

[16] M. Matsui, A. Yamamoto, A. Kuma, Y. Ohsumi, and N. Mizushima, "Organelle degradation during the lens and erythroid differentiation is independent of autophagy," Biochemical and Biophysical Research Communications, vol. 339, no. 2, pp. 485-489, 2006.

[17] K. G. Lyamzaev, O. K. Nepryakhina, V. B. Saprunova et al., "Novel mechanism of elimination of malfunctioning mitochondria (mitoptosis): formation of mitoptotic bodies 
and extrusion of mitochondrial material from the cell," Biochimica et Biophysica Acta, vol. 1777, no. 7-8, pp. 817-825, 2008.

[18] D. Narendra, A. Tanaka, D. F. Suen, and R. J. Youle, "Parkin is recruited selectively to impaired mitochondria and promotes their autophagy," Journal of Cell Biology, vol. 183, no. 5, pp. 795-803, 2008.

[19] C. Vives-Bauza, C. Zhou, Y. Huang et al., "PINK1-dependent recruitment of Parkin to mitochondria in mitophagy," Proceedings of the National Academy of Sciences of the United States of America, vol. 107, no. 1, pp. 378-383, 2010.

[20] P. Anglade, S. Vyas, F. Javoy-Agid et al., "Apoptosis and autophagy in nigral neurons of patients with Parkinson's disease," Histology and Histopathology, vol. 12, no. 1, pp. 2531, 1997.

[21] J. H. Zhu, F. Guo, J. Shelburne, S. Watkins, and C. T. Chu, "Localization of phosphorylated ERK/MAP kinases to mitochondria and autophagosomes in lewy body diseases," Brain Pathology, vol. 13, no. 4, pp. 473-481, 2003.

[22] P. I. Moreira, S. L. Siedlak, X. Wang et al., "Autophagocytosis of mitochondria is prominent in Alzheimer disease," Journal of Neuropathology and Experimental Neurology, vol. 66, no. 6, pp. 525-532, 2007.

[23] J. H. Zhu, S. M. Kulich, T. D. Oury, and C. T. Chu, "Cytoplasmic aggregates of phosphorylated extracellular signalregulated protein kinases in lewy body diseases," American Journal of Pathology, vol. 161, no. 6, pp. 2087-2098, 2002.

[24] G. Perry, H. Roder, A. Nunomura et al., "Activation of neuronal extracellular receptor kinase (ERK) in Alzheimer disease links oxidative stress to abnormal phosphorylation," NeuroReport, vol. 10, no. 11, pp. 2411-2415, 1999.

[25] K. E. Larsen, E. A. Fon, T. G. Hastings, R. H. Edwards, and D. Sulzer, "Methamphetamine-induced degeneration of dopaminergic neurons involves autophagy and upregulation of dopamine synthesis," Journal of Neuroscience, vol. 22, no. 20, pp. 8951-8960, 2002.

[26] R. Castino, G. Lazzeri, P. Lenzi et al., "Suppression of autophagy precipitates neuronal cell death following low doses of methamphetamine," Journal of Neurochemistry, vol. 106, no. 3, pp. 1426-1439, 2008.

[27] C. Gomez-Santos, I. Ferrer, A. F. Santidrián, M. Barrachina, J. Gil, and S. Ambrosio, "Dopamine induces autophagic cell death and $\alpha$-synuclein increase in human neuroblastoma SHSY5Y cells," Journal of Neuroscience Research, vol. 73, no. 3, pp. 341-350, 2003.

[28] S. Dadakhujaev, H. S. Noh, E. J. Jung et al., "Autophagy protects the rotenone-induced cell death in $\alpha$-synuclein overexpressing SH-SY5Y cells," Neuroscience Letters, vol. 472, no. 1, pp. 47-52, 2010.

[29] M. Niso-Santano, J. M. B.-S. Pedro, R. Gómez-Sánchez et al., "ASK1 overexpression accelerates paraquat-induced autophagy via endoplasmic reticulum stress," Toxicological Sciences, vol. 119, no. 1, pp. 156-168, 2011.

[30] K. C. Choi, S. H. Kim, J. Y. Ha, S. T. Kim, and J. H. Son, "A novel mTOR activating protein protects dopamine neurons against oxidative stress by repressing autophagy related cell death," Journal of Neurochemistry, vol. 112, no. 2, pp. 366376, 2010.

[31] A. Petiot, E. Ogier-Denis, E. F. C. Blommaart, A. J. Meijer, and P. Codogno, "Distinct classes of phosphatidylinositol 3'-kinases are involved in signaling pa7thways that control macroautophagy in HT-29 cells," Journal of Biological Chemistry, vol. 275, no. 2, pp. 992-998, 2000.
[32] Y. T. Wu, H. L. Tan, G. Shui et al., "Dual role of 3methyladenine in modulation of autophagy via different temporal patterns of inhibition on class I and III phosphoinositide 3-kinase," Journal of Biological Chemistry, vol. 285, no. 14, pp. 10850-10861, 2010.

[33] L. H. P. Caro, P. J. A. M. Plomp, E. J. Wolvetang, C. Kerkhof, and A. J. Meijer, "3-Methyladenine, a inhibitor of autophagy, has multiple effects on metabolism," European Journal of Biochemistry, vol. 175, no. 2, pp. 325-329, 1988.

[34] S. Pattingre, A. Tassa, X. Qu et al., "Bcl-2 antiapoptotic proteins inhibit Beclin 1-dependent autophagy," Cell, vol. 122, no. 6, pp. 927-939, 2005.

[35] Y. Zhong, Q. J. Wang, X. Li et al., "Distinct regulation of autophagic activity by Atg14L and Rubicon associated with Beclin 1-phosphatidylinositol-3-kinase complex," Nature Cell Biology, vol. 11, no. 4, pp. 468-476, 2009.

[36] K. Matsunaga, T. Saitoh, K. Tabata et al., "Two Beclin 1binding proteins, Atg14L and Rubicon, reciprocally regulate autophagy at different stages," Nature Cell Biology, vol. 11, no. 4, pp. 385-396, 2009.

[37] R. Castino, N. Bellio, C. Follo, D. Murphy, and C. Isidoro, "Inhibition of Pi3k class III-dependent autophagy prevents apoptosis and necrosis by oxidative stress in dopaminergic neuroblastoma cells," Toxicological Sciences, vol. 117, no. 1, pp. 152-162, 2010.

[38] C. T. Chu, J. Zhu, and R. Dagda, "Beclin 1-independent pathway of damage-induced mitophagy and autophagic stress: implications for neurodegeneration and cell death," Autophagy, vol. 3, no. 6, pp. 663-666, 2007.

[39] A. M. Cuervo, L. Stafanis, R. Fredenburg, P. T. Lansbury, and D. Sulzer, "Impaired degradation of mutant $\alpha$-synuclein by chaperone-mediated autophagy," Science, vol. 305, no. 5688, pp. 1292-1295, 2004.

[40] M. Martinez-Vicente, Z. Talloczy, S. Kaushik et al., "Dopamine-modified $\alpha$-synuclein blocks chaperonemediated autophagy," Journal of Clinical Investigation, vol. 118, no. 2, pp. 777-778, 2008.

[41] T. Vogiatzi, M. Xilouri, K. Vekrellis, and L. Stefanis, "Wild type $\alpha$-synuclein is degraded by chaperone-mediated autophagy and macroautophagy in neuronal cells," Journal of Biological Chemistry, vol. 283, no. 35, pp. 23542-23556, 2008.

[42] M. Xilouri, T. Vogiatzi, K. Vekrellis, D. Park, and L. Stefanis, "Abberant $\alpha$-synuclein confers toxicity to neurons in part through inhibition of chaperone-mediated autophagy," PLoS ONE, vol. 4, no. 5, article e5515, 2009.

[43] W. H. Yu, B. Dorado, H. Y. Figueroa et al., "Metabolic activity determines efficacy of macroautophagic clearance of pathological oligomeric $\alpha$-synuclein," American Journal of Pathology, vol. 175, no. 2, pp. 736-747, 2009.

[44] S. J. Chinta, J. K. Mallajosyula, A. Rane, and J. K. Andersen, "Mitochondrial alpha-synuclein accumulation impairs complex I function in dopaminergic neurons and results in increased mitophagy in vivo," Neuroscience Letters, vol. 486, no. 3, pp. 235-239, 2010.

[45] A. R. Winslow, C.-W. Chen, S. Corrochano et al., " $\alpha$ synuclein impairs macroautophagy: implications for Parkinson's disease," Journal of Cell Biology, vol. 190, no. 6, pp. 1023-1037, 2010.

[46] V. N. Pivtoraiko, A. J. Harrington, B. J. Mader et al., "Lowdose bafilomycin attenuates neuronal cell death associated with autophagy-lysosome pathway dysfunction," Journal of Neurochemistry, vol. 114, no. 4, pp. 1193-1204, 2010.

[47] D. MacLeod, J. Dowman, R. Hammond, T. Leete, K. Inoue, and A. Abeliovich, "The familial Parkinsonism gene LRRK2 
regulates neurite process morphology," Neuron, vol. 52, no. 4, pp. 587-593, 2006.

[48] E. D. Plowey, S. J. Cherra, Y. J. Liu, and C. T. Chu, "Role of autophagy in G2019S-LRRK2-associated neurite shortening in differentiated SH-SY5Y cells," Journal of Neurochemistry, vol. 105, no. 3, pp. 1048-1056, 2008.

[49] S. J. Cherra III, S. M. Kulich, G. Uechi et al., "Regulation of the autophagy protein LC3 by phosphorylation," Journal of Cell Biology, vol. 190, no. 4, pp. 533-539, 2010.

[50] J. Alegre-Abarrategui, H. Christian, M. M. P. Lufino et al., "LRRK2 regulates autophagic activity and localizes to specific membrane microdomains in a novel human genomic reporter cellular model," Human Molecular Genetics, vol. 18, no. 21, pp. 4022-4034, 2009.

[51] T. Kitada, A. Pisani, M. Karouani et al., "Impaired dopamine release and synaptic plasticity in the striatum of Parkin-/mice," Journal of Neurochemistry, vol. 110, no. 2, pp. 613-621, 2009.

[52] G. Oyama, K. Yoshimi, S. Natori et al., "Impaired in vivo dopamine release in parkin knockout mice," Brain Research, vol. 1352, no. C, pp. 214-222, 2010.

[53] J. C. Greene, A. J. Whitworth, I. Kuo, L. A. Andrews, M. B. Feany, and L. J. Pallanck, "Mitochondrial pathology and apoptotic muscle degeneration in Drosophila parkin mutants," Proceedings of the National Academy of Sciences of the United States of America, vol. 100, no. 7, pp. 4078-4083, 2003.

[54] D. Chen, F. Gao, B. Li et al., "Parkin mono-ubiquitinates Bcl2 and regulates autophagy," Journal of Biological Chemistry, vol. 285, no. 49, pp. 38214-38223, 2010.

[55] A. Wood-Kaczmar, S. Gandhi, Z. Yao et al., "PINK1 is necessary for long term survival and mitochondrial function in human dopaminergic neurons," PLoS ONE, vol. 3, no. 6, article e2455, 2008.

[56] V. A. Morais, P. Verstreken, A. Roethig et al., "Parkinson's disease mutations in PINK1 result in decreased complex I activity and deficient synaptic function," EMBO Molecular Medicine, vol. 1, no. 2, pp. 99-111, 2009.

[57] A. Kathrin Lutz, N. Exner, M. E. Fett et al., "Loss of parkin or PINK1 function increases Drp1-dependent mitochondrial fragmentation," Journal of Biological Chemistry, vol. 284, no. 34, pp. 22938-22951, 2009.

[58] S. Michiorri, V. Gelmetti, E. Giarda et al., "The Parkinsonassociated protein PINK1 interacts with Beclin1 and promotes autophagy," Cell Death and Differentiation, vol. 17, no. 6, pp. 962-974, 2010.

[59] S. Kawajiri, S. Saiki, S. Sato et al., "PINK1 is recruited to mitochondria with parkin and associates with LC3 in mitophagy," FEBS Letters, vol. 584, no. 6, pp. 1073-1079, 2010.

[60] E. Ziviani, R. N. Tao, and A. J. Whitworth, "Drosophila Parkin requires PINK1 for mitochondrial translocation and ubiquitinates Mitofusin," Proceedings of the National Academy of Sciences of the United States of America, vol. 107, no. 11, pp. 5018-5023, 2010.

[61] A. J. Whitworth, J. R. Lee, V. M. W. Ho, R. Flick, R. Chowdhury, and G. A. McQuibban, "Rhomboid-7 and HtrA2/Omi act in a common pathway with the Parkinson's disease factors Pink1 and Parkin," Disease Models and Mechanisms, vol. 1, no. 2-3, pp. 168-174, 2008.

[62] D. P. Narendra, S. M. Jin, A. Tanaka et al., "PINK1 is selectively stabilized on impaired mitochondria to activate Parkin,” PLoS Biology, vol. 8, no. 1, Article ID e1000298, 2010.
[63] N. Matsuda, S. Sato, K. Shiba et al., "PINK1 stabilized by mitochondrial depolarization recruits Parkin to damaged mitochondria and activates latent Parkin for mitophagy," Journal of Cell Biology, vol. 189, no. 2, pp. 211-221, 2010.

[64] I. Irrcher, H. Aleyasin, E. L. Seifert et al., "Loss of the Parkinson's disease-linked gene DJ-1 perturbs mitochondrial dynamics," Human Molecular Genetics, vol. 19, no. 19, pp. 3734-3746, 2010.

[65] K. J. Thomas, M. K. McCoy, J. Blackinton et al., "DJ-1 acts in parallel to the PINK1/parkin pathway to control mitochondrial function and autophagy," Human Molecular Genetics, vol. 20, no. 1, pp. 40-50, 2011.

[66] E. Ogier-Denis, S. Pattingre, J. El Benna, and P. Codogno, "Erk1/2-dependent phosphorylation of $\mathrm{G} \alpha$-interacting protein stimulates its GTPase accelerating activity and autophagy in human colon cancer cells," Journal of Biological Chemistry, vol. 275, no. 50, pp. 39090-39095, 2000.

[67] J. Wang, M. W. Whiteman, H. Lian et al., "A non-canonical MEK/ERK signaling pathway regulates autophagy via regulating Beclin 1," Journal of Biological Chemistry, vol. 284, no. 32, pp. 21412-21424, 2009.

[68] R. A. González-Polo, M. Niso-Santano, J. M. Morán et al., "Silencing DJ-1 reveals its contribution in paraquat-induced autophagy," Journal of Neurochemistry, vol. 109, no. 3, pp. 889-898, 2009.

[69] C. He and D. J. Klionsky, "Regulation mechanisms and signaling pathways of autophagy," Annual Review of Genetics, vol. 43, pp. 67-93, 2009.

[70] I. Kissova, M. Deffieu, S. Manon, and N. Camougrand, "Uth1p is involved in the autophagic degradation of mitochondria," Journal of Biological Chemistry, vol. 279, no. 37, pp. 39068-39074, 2004.

[71] R. Tal, G. Winter, N. Ecker, D. J. Klionsky, and H. Abeliovich, "Auplp, a yeast mitochondrial protein phosphatase homolog, is required for efficient stationary phase mitophagy and cell survival," Journal of Biological Chemistry, vol. 282, no. 8, pp. 5617-5624, 2007.

[72] T. Kanki, K. Wang, Y. Cao, M. Baba, and D. J. Klionsky, "Atg32 is a mitochondrial protein that confers selectivity during mitophagy," Developmental Cell, vol. 17, no. 1, pp. 98-109, 2009.

[73] I. Kim and J. J. Lemasters, "Mitophagy selectively degrades individual damaged mitochondria after photoirradiation," Antioxidants \& Redox Signaling. In press.

[74] K. Obara and Y. Ohsumi, "Dynamics and function of PtdIns(3)P in autophagy," Autophagy, vol. 4, no. 7, pp. 952954, 2008.

[75] F. Scarlatti, R. Maffei, I. Beau, P. Codogno, and R. Ghidoni, "Role of non-canonical Beclin 1-independent autophagy in cell death induced by resveratrol in human breast cancer cells," Cell Death and Differentiation, vol. 15, no. 8, pp. 13181329, 2008.

[76] K. S. Yee, S. Wilkinson, J. James, K. M. Ryan, and K. H. Vousden, "PUMA- and Bax-induced autophagy contributes to apoptosis," Cell Death and Differentiation, vol. 16, no. 8, pp. 1135-1145, 2009.

[77] S. Tian, J. Lin, J. Zhou et al., "Beclin 1-independent autophagy induced by a Bcl-XL/Bcl-2 targeting compound, Z18," Autophagy, vol. 6, no. 8, pp. 1032-1041, 2010.

[78] J. Y. Lee, H. Koga, Y. Kawaguchi et al., "HDAC6 controls autophagosome maturation essential for ubiquitin-selective quality-control autophagy," EMBO Journal, vol. 29, no. 5, pp. 969-980, 2010. 
[79] A. Iwata, B. E. Riley, J. A. Johnston, and R. R. Kopito, "HDAC6 and microtubules are required for autophagic degradation of aggregated Huntingtin," Journal of Biological Chemistry, vol. 280, no. 48, pp. 40282-40292, 2005.

[80] S. Pankiv, T. H. Clausen, T. Lamark et al., "p62/SQSTM1 binds directly to Atg8/LC3 to facilitate degradation of ubiquitinated protein aggregates by autophagy," Journal of Biological Chemistry, vol. 282, no. 33, pp. 24131-24145, 2007.

[81] J. M. M. Tan, E. S. P. Wong, V. L. Dawson, T. M. Dawson, and K. L. Lim, "Lysine 63-linked polyubiquitin potentially partners with p62 to promote the clearance of protein inclusions by autophagy," Autophagy, vol. 4, no. 2, pp. 251253, 2008.

[82] T. Lamark, V. Kirkin, I. Dikic, and T. Johansen, "NBR1 and p62 as cargo receptors for selective autophagy of ubiquitinated targets," Cell Cycle, vol. 8, no. 13, pp. 1986-1990, 2009.

[83] I. Novak, V. Kirkin, D. G. McEwan et al., "Nix is a selective autophagy receptor for mitochondrial clearance," $E M B O$ Reports, vol. 11, no. 1, pp. 45-51, 2010.

[84] I. Kim, S. Rodriguez-Enriquez, and J. J. Lemasters, "Selective degradation of mitochondria by mitophagy," Archives of Biochemistry and Biophysics, vol. 462, no. 2, pp. 245-253, 2007.

[85] J. J. Lemasters, A. L. Nieminen, T. Qian et al., "The mitochondrial permeability transition in cell death: a common mechanism in necrosis, apoptosis and autophagy," Biochimica et Biophysica Acta, vol. 1366, no. 1-2, pp. 177-196, 1998.

[86] S. Rodriguez-Enriquez, I. Kim, R. T. Currin, and J. J. Lemasters, "Tracker dyes to probe mitochondrial autophagy (mitophagy) in rat hepatocytes," Autophagy, vol. 2, no. 1, pp. 39-46, 2006.

[87] C. T. Chu, "A pivotal role for PINK1 and autophagy in mitochondrial quality control: implications for Parkinson disease," Human Molecular Genetics, vol. 19, no. 1, pp. R28R37, 2010.

[88] W. Lin and U. J. Kang, "Characterization of PINK1 processing, stability, and subcellular localization," Journal of Neurochemistry, vol. 106, no. 1, pp. 464-474, 2008.

[89] M. E. Gegg, J. M. Cooper, K.-Y. Chau, M. Rojo, A. H. V. Schapira, and J.-W. Taanman, "Mitofusin 1 and mitofusin 2 are ubiquitinated in a PINK1/parkin-dependent manner upon induction of mitophagy," Human Molecular Genetics, vol. 19, no. 24, pp. 4861-4870, 2010.

[90] S. Geisler, K. M. Holmström, D. Skujat et al., "PINK1/Parkinmediated mitophagy is dependent on VDAC1 and p62/SQSTM1," Nature Cell Biology, vol. 12, no. 2, pp. $119-131,2010$.

[91] J. Y. Lee, Y. Nagano, J. P. Taylor, K. L. Lim, and T. P. Yao, "Disease-causing mutations in Parkin impair mitochondrial ubiquitination, aggregation, and HDAC6-dependent mitophagy," Journal of Cell Biology, vol. 189, no. 4, pp. 671679,2010

[92] C. Schwarzer, S. Barnikol-Watanabe, F. P. Thinnes, and N. Hilschmann, "Voltage-dependent anion-selective channel (VDAC) interacts with the dynein light chain Tctex1 and the heat-shock protein PBP74," International Journal of Biochemistry and Cell Biology, vol. 34, no. 9, pp. 1059-1070, 2002.

[93] D. P. Narendra, L. A. Kane, D. N. Hauser, I. M. Fearnley, and R. J. Youle, "p62/SQSTM1 is required for Parkin-induced mitochondrial clustering but not mitophagy; VDAC1 is dispensable for both," Autophagy, vol. 6, no. 8, pp. 1090$1106,2010$.
[94] J. Zhang and P. A. Ney, "Autophagy-dependent and -independent mechanisms of mitochondrial clearance during reticulocyte maturation," Autophagy, vol. 5, no. 7, pp. 10641065, 2009.

[95] N. Exner, B. Treske, D. Paquet et al., "Loss-of-function of human PINK1 results in mitochondrial pathology and can be rescued by parkin," Journal of Neuroscience, vol. 27, no. 45, pp. 12413-12418, 2007.

[96] I. E. Clark, M. W. Dodson, C. Jiang et al., "Drosophila pink1 is required for mitochondrial function and interacts genetically with parkin," Nature, vol. 441, no. 7097, pp. 11621166, 2006.

[97] A. Rakovic, A. Grünewald, P. Seibler et al., "Effect of endogenous mutant and wild-type PINK1 on Parkin in fibroblasts from Parkinson disease patients," Human Molecular Genetics, vol. 19, no. 16, pp. 3124-3137, 2010.

[98] J. M. Rose, S. S. Novoselov, P. A. Robinson, and M. E. Cheetham, "Molecular chaperone-mediated rescue of mitophagy by a Parkin RING1 domain mutant," Human Molecular Genetics, vol. 20, no. 1, pp. 16-27, 2011.

[99] R. A. Kirkland, R. M. Adibhatla, J. F. Hatcher, and J. L. Franklin, "Loss of cardiolipin and mitochondria during programmed neuronal death: evidence of a role for lipid peroxidation and autophagy," Neuroscience, vol. 115, no. 2, pp. 587-602, 2002.

[100] Y. Gu, C. Wang, and A. Cohen, "Effect of IGF-1 on the balance between autophagy of dysfunctional mitochondria and apoptosis," FEBS Letters, vol. 577, no. 3, pp. 357-360, 2004.

[101] M. Alemi, A. Prigione, A. Wong et al., "Mitochondrial DNA deletions inhibit proteasomal activity and stimulate an autophagic transcript," Free Radical Biology and Medicine, vol. 42, no. 1, pp. 32-43, 2007.

[102] G. Twig, A. Elorza, A. J. A. Molina et al., "Fission and selective fusion govern mitochondrial segregation and elimination by autophagy," EMBO Journal, vol. 27, no. 2, pp. 433-446, 2008.

[103] K. E. Miller and M. P. Sheetz, "Axonal mitochondrial transport and potential are correlated," Journal of Cell Science, vol. 117, no. 13, pp. 2791-2804, 2004.

[104] V. S. van Laar, B. Arnold, S. J. Cassady, C. T. Chu, E. A. Burton, and S. B. Berman, "Bioenergetics of neurons inhibit the translocation response of Parkin following rapid mitochondrial depolarization," Human Molecular Genetics, vol. 20, no. 5, pp. 927-940, 2011. 


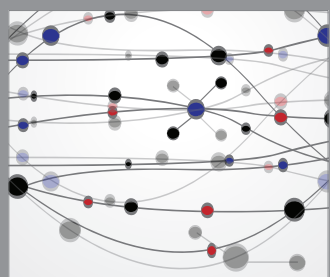

The Scientific World Journal
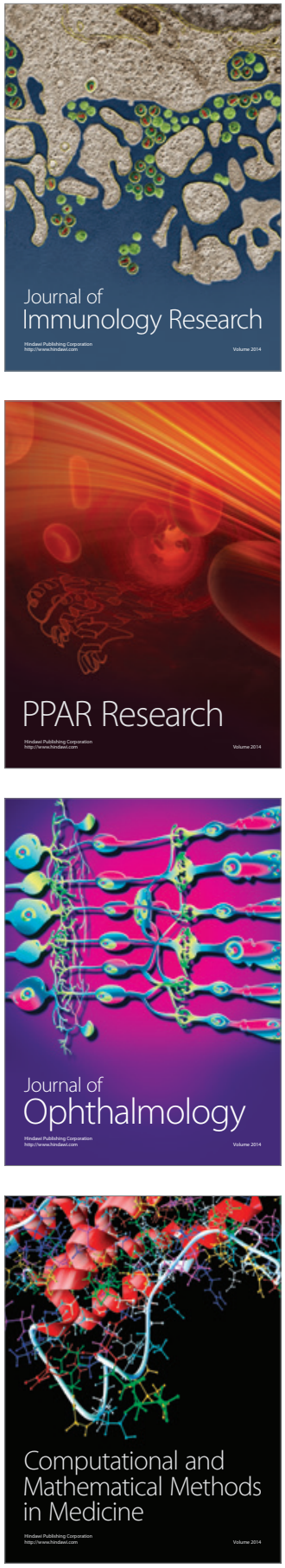

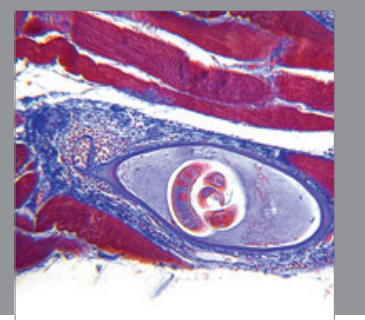

Gastroenterology

Research and Practice
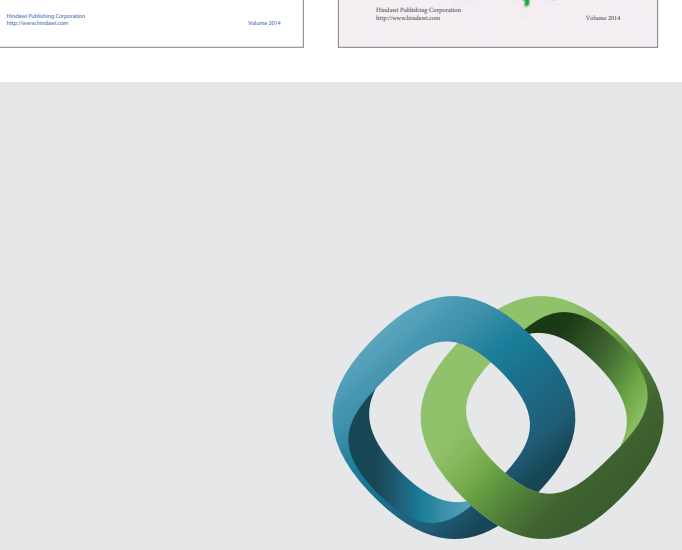

\section{Hindawi}

Submit your manuscripts at

http://www.hindawi.com
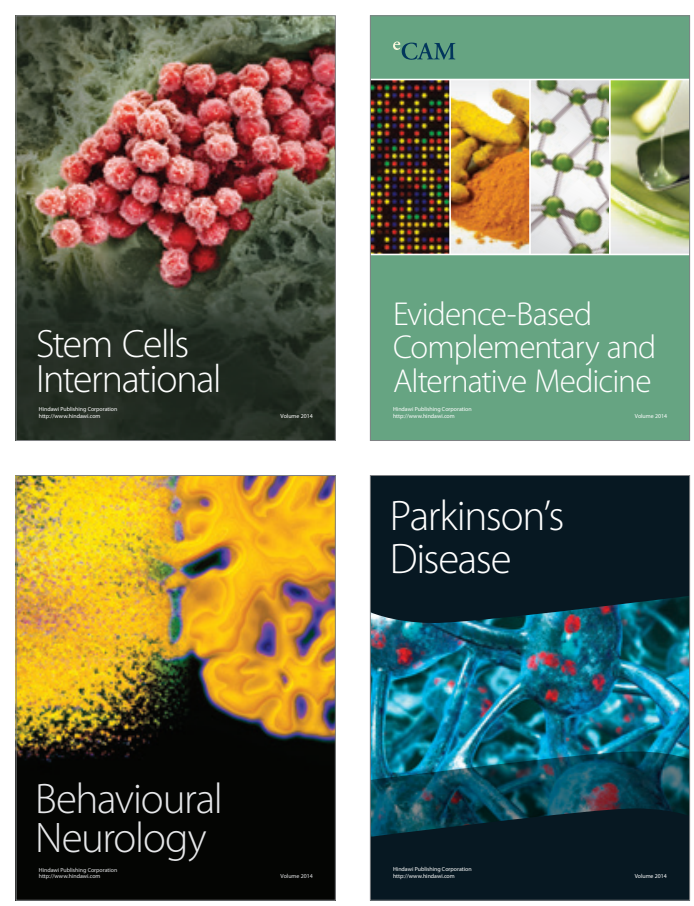

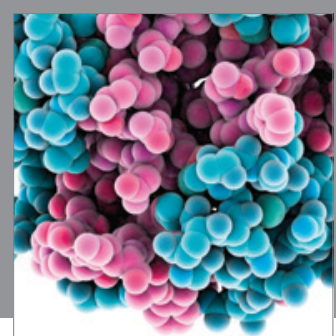

Journal of
Diabetes Research

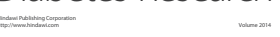

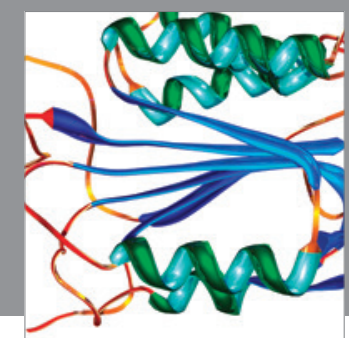

Disease Markers
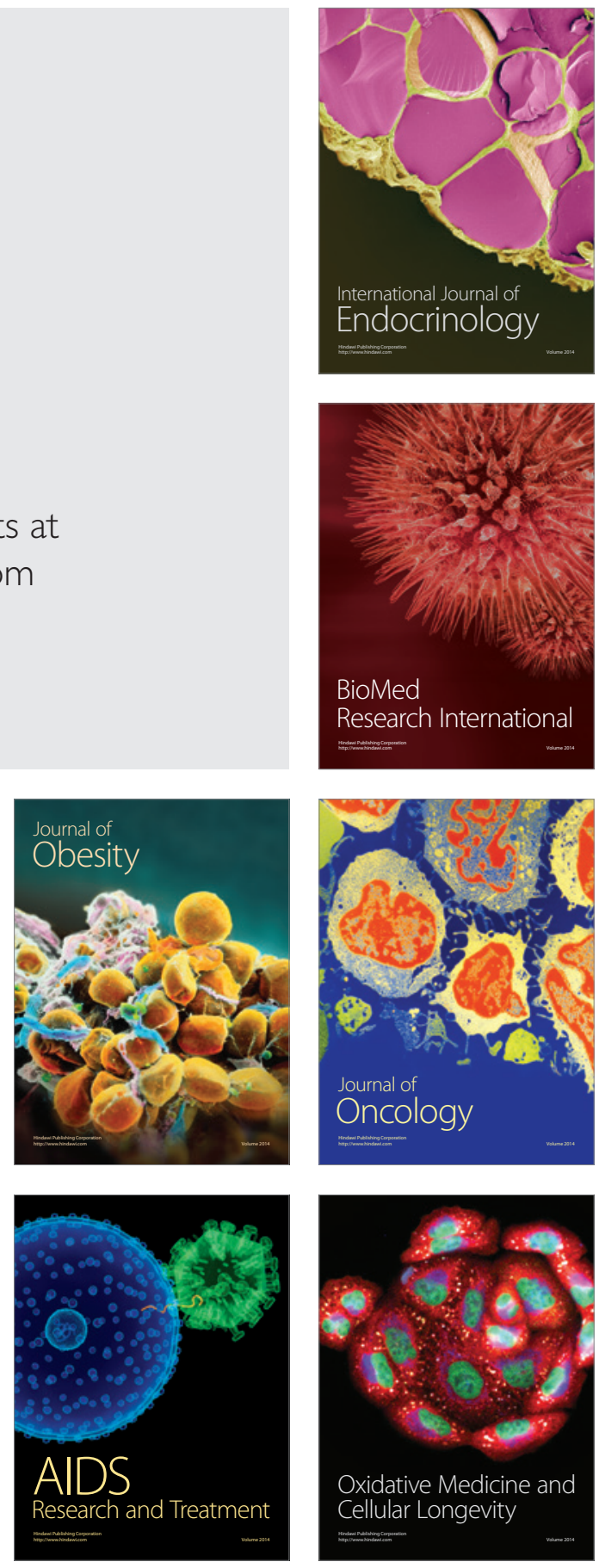